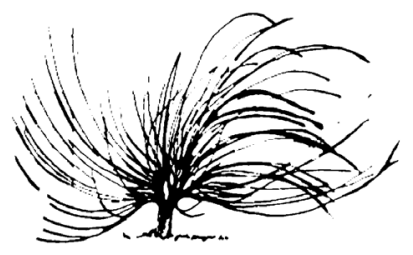

\title{
Juego y gamificación: Innovación educativa en una sociedad en continuo cambio
}

\author{
Javier Gil Quintana ${ }^{1}$ \\ Universidad Nacional de Educación a Distancia \\ España \\ jgilquintana@invi.uned.es \\ Elizabeth Prieto Jurado ${ }^{2}$ \\ Universidad Nacional de Educación a Distancia \\ España \\ eliprijur@hotmail.com
}

\begin{abstract}
Resumen
En este ensayo hemos pretendido realizar una aproximación al concepto de juego y de gamificación, al ser una propuesta innovadora que se está potencializando en la actualidad en las prácticas educativas que están incorporando experiencias de gamificación en sus aulas. Basándonos en diferentes estudios que a lo largo de la historia han reflexionado acerca del papel de la educación $\mathrm{y}$, partiendo de la corriente pedagógica de la escuela nueva, apoyamos su premisa sobre la importancia del
\end{abstract}

Recibido: 10 de noviembre de 2018. Aprobado: 10 de abril de 2019.

http://dx.doi.org/10.15359/rep.14-1.5

1 Dr. Javier Gil Quintana. Doctor en educación y comunicación, premio extraordinario. Máster de Educación y Comunicación en la Red por la UNED. Máster en Tecnologías Digitales y Sociedad del Conocimiento. Experto de análisis de medios, sistemas interactivos de comunicación y software libre. Maestro de educación infantil y primaria. Profesor contratado doctor de la Universidad Católica de Ávila y colaborador de la UNED. Sus líneas de investigación son MOOC, transmedia, aprendizaje digital y gamificación.

2 Lda. Elizabeth Prieto Jurado. Doctoranda en educación y comunicación. Máster de Educación y Comunicación en la Red por la UNED. Licenciada en Psicopedagogía. Maestra de lengua extranjera (inglés). Sus líneas de investigación son aprendizaje digital y gamificación. 
juego en el aprendizaje; confirmamos, también, un gran abanico de ventajas que actualmente tiene gamificación y su integración en el aprendizaje digital, al posibilitar el aumento de la participación estudiantil en la construcción colectiva del conocimiento, la proyección de la interacción en el aula, una mayor motivación hacia el aprendizaje y un carácter más divertido en las experiencias de aula, con el fin de comprometerse en el alcance y el superación de las líneas curriculares marcadas para su edad y nivel correspondiente.

Palabras clave: Aprendizaje activo, aprendizaje colaborativo, comunicación educativa, gamificación.

\begin{abstract}
In this essay, we have tried to make an approximation to the concept of game and gamification since it is an innovative proposal that is currently being used in educational practices whose teachers are incorporating experiences of gamification in their classrooms. Based on the different authors that throughout history have reflected and concentrated on the role of education which started from the pedagogical methods of the new, current school of thought, we support its premise on the importance of implementing game in learning, also confirming a wide range of advantages that gamification and its integration in digital learning currently have, making it possible to increase the participation of students in the collective construction of knowledge, the projection of its interaction in the classroom, a greater motivation towards learning, and a more enriching and fun environment in the classroom and its experiences with the aim of engaging in the achievement and exceeding of the curricular lines set for their corresponding age and level.
\end{abstract}

Keywords: active learning, collaborative learning, educational communication, gamification 


\section{Introducción}

Para adentrarnos de lleno en el análisis del contexto educativo que pretende nuestro ensayo realizado por docentes desde una perspectiva de innovación educativa, debemos analizar el entorno social en el que este se desarrolla y qué tipo de individuos forman parte de él. Por ello, iremos de lo general a lo particular, partiendo de un análisis de la sociedad líquida en la que nos encontramos sumergidos, siguiendo con una exposición de las características de las generaciones protagonistas de los cambios que estamos experimentando, así como el entorno educativo en el que crecen y se desarrollan como individuos capaces de formar parte de una ciudadanía activa y desenvolverse de manera autónoma. Abordaremos el poder del conocimiento en la sociedad de la comunicación y cómo esta exige ciertas destrezas que deben ser adquiridas desde los primeros años de vida. Seguidamente analizaremos el sector educativo al que estarán dirigidas las prácticas innovadoras que ya han sido aplicadas y las que se crearán a medida que vayamos tomando conciencia de en qué consiste el cambio educativo.

Desde escenarios digitales y analógicos que convergen en una misma realidad educativa donde convive la generación digital (Gil-Quintana, 2015), se trata de responder a la pregunta: ¿qué motiva a la joven ciudadanía?

Este sector de la sociedad presente vive por las redes sociales y para estas, interactuando de forma continuada y descubriéndose en el mundo como una ciudadanía hiperconectada que se encamina hacia un futuro asombroso a nivel de comunicación. A este alumnado digital el sistema educativo debe responder con el gran reto para motivarlo. Una vez aquí, analizaremos el tipo de motivación que existe y puede poseer este estudiantado, así como las diferentes teorías relacionadas con la innovación educativa, tales como el conductismo, cognitivismo, constructivismo y conectivismo, diferentes teorías que explican la adquisición de aprendizaje de diversas formas.

Todo esto estará unido al tratamiento habitual, dentro de las prácticas innovadoras, del juego como agente, aunque no el único, para mejorar la motivación del alumnado que alberga nuestras aulas. Por último, será necesario afrontar la transición en los centros educativos a medida que se han ido aconteciendo estos cambios y han venido a formar parte de ellos diversas generaciones con sus características propias. 
¿Puede la tecnología ayudarnos a motivar a nuestro alumnado? Hablar de educación en esta sociedad hiperconectada es hablar necesariamente de la integración de la tecnología como objeto de estudio y como recurso didáctico, al tratarse de unos medios con gran potencialidad. Dentro de este ámbito de innovación, veremos la relación estrecha que van a forjar gamificación y tecnología, donde esta última será el elemento vehicular a través del cual se desarrollarán y pondrán en práctica muchos de los sistemas gamificados.

\section{El poder del conocimiento en una sociedad en profundo cambio}

La sociedad actual tiene como característica principal su carácter cambiante, experimenta, de forma continua, transformaciones (Tedesco, 1999) y aduce un valor cuasi fundamental a la información mediante su recepción, búsqueda, organización, almacenamiento y aplicación en las diferentes situaciones de la vida cotidiana. Esta realidad líquida tiene su proyección más fuerte a través de las tecnologías digitales (Colás, 2003), un contexto social definido como la sociedad del conocimiento, término utilizado para describir las transformaciones sociales que se están produciendo en la sociedad moderna, "en el ámbito de planificación de la educación y formación, en el ámbito de la organización (gestión de conocimiento) y del trabajo (trabajo de conocimiento)" (Krüguer, 2006, p. 4). Unido a esta definición, podemos encontrar otras referencias condicionadas por el enfoque que se aborde desde el ámbito educativo, social, empresarial, tecnológico o ambiental. Lo cierto es que no existe una noción clara sobre lo que es en realidad la sociedad del conocimiento, por lo cual se crean, en ocasiones, confusiones con conceptos como sociedad red (Castells, 2000) y sociedad de la información.

En nuestro caso concreto, relacionado con el ámbito educativo, el valor más apreciado será la construcción del conocimiento y no la simple recepción o elaboración de información; mediante esta edificación se pueden resolver problemas con un enfoque colaborativo, sistémico y ético, en busca de la realización personal de los individuos en la medida que contribuyen al tejido social. Se trata, entonces, del desarrollo tanto del individuo como de la sociedad en general y el ambiente de construcción, ya que cuando una persona se fortalece de algo creado, las demás también se desarrollan dentro de un modelo pedagógico interactivo, una comunicación horizontal y bidireccional (García y Godínez, 2015). 
El concepto de sociedad del conocimiento se diferencia de sociedad red y sociedad de la información (Krüger, 2006). Entendemos por sociedad red (Castells, 2000), aquellas comunidades que se dedican a interconectar información que se procesa, almacena y transmite sin inconvenientes de tiempo, distancia, volumen a través de medios tecnológicos. Es la sociedad cuya estructura está construida en torno a redes de información a partir de la tecnología de información estructurada en internet. Pero internet no es simplemente una tecnología, es el medio de comunicación que constituye la forma organizativa de nuestra sociedad, es el equivalente a lo que fue la factoría en la era industrial o la gran corporación en la era empresarial; es el corazón de un nuevo paradigma sociotécnico que constituye en realidad la base material de nuestras vidas y de nuestras formas de relación, de trabajo y de comunicación. Lo que hace internet es procesar la virtualidad y transformarla en nuestra realidad, constituyendo la sociedad red, que es la sociedad en que vivimos.

La sociedad de la información es expresión de las realidades y capacidades de los medios de comunicación más nuevos, o renovados merced a los desarrollos tecnológicos que se consolidaron en la última década del siglo: la televisión, el almacenamiento de información, la propagación de video, sonido y textos han podido comprimirse en soportes de almacenamiento como los discos compactos o a través de señales que no podrían conducir todos esos datos, si no hubieran sido traducidos a formatos digitales. La digitalización de la información es el sustento de la nueva revolución comunicativa, su expresión, hasta ahora muy compleja, seguirá desarrollándose para asumir nuevos formatos en medio plazo. La sociedad del conocimiento se diferencia de la sociedad red porque trasciende la tecnología, se desarrolla a través de diferentes medios y afronta los problemas del contexto. Por otro lado, la sociedad del conocimiento difiere de la sociedad de la información porque se orienta a la gestión, comprensión y cocreación del conocimiento para resolver los problemas (Tobón, 2013, 2014), así, trasciende la información. Sociedad del conocimiento debe ser la apuesta desde las aulas, esto es, espacios que sirvan para construir colectivamente, convirtiendo las tecnologías de la información y conocimiento -TIC- en tecnologías de la relación, información y comunicación -TRIC- (Marta-Lazo y Gabelas-Barroso, 2016) y tecnologías del aprendizaje y el conocimiento -TAC-. No podemos continuar basando nuestras prácticas pedagógicas 
en la transmisión de información, sino que nuestro propósito tiene que ser hacer que nuestro alumnado construya, no reproduzca, fomentando así la construcción colectiva del conocimiento.

Las diversas generaciones han tenido siempre su protagonismo en la evolución de la humanidad, pero el momento actual parece atribuírsele a la joven generación del tercer milenio. Se nos antoja interesante echar un vistazo a este sector de población, ya que, además de ser el grupo receptor de las prácticas educativas del sistema educativo actual, sus formas de vida, de relacionarse con sus semejantes y aprender están muy relacionadas con los dispositivos electrónicos, las redes sociales, la narrativa digital, los videojuegos y la gamificación.

Las características principales de este grupo etario han sido estudiadas por varias investigaciones. A finales del siglo pasado, Tapscott (1998) ya indicaba que la generación internet aprende, juega y se comunica en forma muy distinta a la de sus grupos primogénitos. Iniciado el siglo XXI, Beck y Wade (2004) afirmaban que la generación de personas jugadoras tiene sistemáticamente diferentes formas de trabajar que son la consecuencia de un factor central: crecer con los videojuegos. Más próximo a nuestros días se considera la juventud, catalogada, entre otros términos, como generación Y o millennials, que se refiere al conjunto de personas nacidas entre los años ochenta y el principio del siglo XXI, queda atrás la generación X (personas nacidas tras la generación del baby boom, entre principios de los años 60 y mediados de los 70) de la que son generaciones sucesoras. Este término hace referencia a una clasificación basada en la relación de su vida cotidiana con la tecnología a través de la cual se comunican, relacionan, consumen y comparten en sus espacios de ocio y formativos. Nadie puede dudar que la juventud se encuentra sometida a una incontable cantidad de estímulos procedentes de diversas fuentes (Guevara, 2015) o múltiples pantallas. Son personas que tienen una gran capacidad para adaptarse continuamente a los cambios de una sociedad que se ha proyectado cambiante o líquida y en relativamente escaso periodo de tiempo. Tienen una forma muy distinta de ver el mundo, las estructuras sociales, las relaciones entre personas $\mathrm{y}$, a pesar de ello, son una de las generaciones mejor preparadas académicamente. Son jóvenes que consideran que deben sobrepasar a sus progenitores o progenitoras, a quienes necesitan para sobrevivir a nivel económico, pero se encuentran con la imposibilidad de hacerlo, en un 
mundo de trabajos volátiles y desempleo persistente, proyectos que nacen muertos y esperanzas frustradas (Bauman, 2013).

A pesar de las distintas afirmaciones que podamos hacer desde el pensamiento docente, se tiene una información muy mínima y controvertida, que puede conducir al error de generalizar las características propias de nuestro alumnado. Ciertos estudios enfocan a la joven ciudadanía como narcisista y vaga (Stein, 2013); es etiquetada como digital, multipantalla y multidispositivo, crítica, sociable y con una alta exigencia en cuanto a consumo y vida se refiere (García, Tur-Viñes, y Pastor, 2018; Gutiérrez-Rubí, 2015); otros estudios se centran en el ámbito de su autoestima catalogándola de baja debido a falsas perspectivas y promesas por parte de sus familias (Sinek, 2017); también la etiquetan como la generación Peter Pan (Semana, 2017); y, en contra, otros grupos con una perspectiva más esperanzadora, aseguran que serán la generación que cambiará el mundo (Fernández, 2012).

En otra línea de pensamiento y relacionado con la comunicación, la $B B C$ el 23 de febrero de 2018 señalaba en la noticia "Creo que nunca hice una llamada de teléfono: Cómo se comunica la generación muda" que el psicólogo Dimitrios Tsivrikos, del University College de Londres, la llama generación muda, debido a que prefieren mandar mensajes de texto y utilizar las redes sociales para comunicarse en lugar de realizar llamadas. De lo que no podemos dudar es de que es una juventud nacida y educada en ambientes altamente tecnologizados, con una fuerte cultura cliente-servicio y, en consonancia con ella, se presenta la educación como una mercancía para ser adquirida y consumida. Por eso esperan que su acercamiento al conocimiento sea lo más rápido, entretenido y sencillo posible, al tiempo que buscan maximizar la relación tiempo de estudio / resultados obtenidos: aspiran a reducir lo más posible el primero al tiempo que incrementan los segundos. Son jóvenes que prefieren la práctica a la teoría, las tareas grupales a las individuales y la información en formato digital a los libros analógicos. Las personas millennials poseen escasa habilidad para resolver problemas, para seguir una argumentación o una demostración, debido principalmente a que muestran dificultades para tener presentes los pasajes lógicos de un desarrollo hasta llegar a la conclusión, presentan, además, dificultades para planificar a largo plazo.

Desde el ámbito docente podemos comprobar cómo los dispositivos electrónicos tienen una presencia creciente en su vida, dedican 
gran cantidad de tiempo a jugar con estos, disfrutan con la creación de videos o ilustraciones digitales, comparten fotos propias en las redes sociales, utilizan su imaginación gracias a aplicaciones de edición que posibilitan el desarrollo de la cultura de la remezcla o remix (Lessig, 2012). Hemos podido observar cómo se abren paso en el espacio digital proyectándose como youtubers, que opinan sobre hechos actuales o acerca de experiencias de ludificación en videojuegos, se presentan también como bookstagrammers, lectores o lectoras que aman fotografiar sus libros de lectura y hacen uso de estrategias de marketing publicitario con la finalidad de despertar el deseo de leer en su público seguidor (Gil-Quintana, 2016a). Un alto porcentaje de estos grupo de jóvenes son gamers, de ahí que se les denomine también generación G, pues utilizan sus dispositivos electrónicos como soporte principal para jugar, por lo que hacen que los sistemas gamificados sean cada vez más efectivos. Todo un entramado de creación que busca no solo compartir con la ciudadanía digital, sino también convertirse en influencers que se publiciten más allá de sus entornos sociales más próximos. Estos juguetes sociales que se han convertido en el deseo de posesión desde la infancia; son cada vez más una necesidad para la ciudadanía en esta edad infantil y adolescente.

Hemos podido comprobar cómo estos grupos muestran interés por seguir a sus youtubers de preferencia o influencers, según los datos ofrecidos en la investigación Transalfabetismos. Competencias transmedia y estrategias informales de aprendizaje de los adolescentes (Universitat Pompeu Fabra, s. f.) $)^{3}$, en la cual hemos tenido el gusto de participar. Esta se llevó a cabo en diferentes contextos sociales, geográficos y culturales de cinco comunidades autónomas (Cataluña, Comunidad Valenciana, Andalucía, Madrid y Galicia). Los resultados del estudio ponen de manifiesto también su motivación por conversar con sus pares del mismo colegio a través de grupos de WhatsApp, en redes sociales como Instagram, en comunidades virtuales afines a ciertos videojuegos o hobbies, con sus colegas del barrio y familiares con quienes establecen algunos lazos comunicativos en la red.

3 Proyecto financiado por el Programa Estatal de Investigación, Desarrollo e Innovación Orientada a los Retos de la Sociedad, en el marco del Plan Estatal de Investigación Científica y Técnica y de Innovación 2013-2016 - Ref. CSO2014-56250-R. Disponible en https:// www.upf.edu/web/medium/projectes-de recerca//asset_publisher/hNFMvM6Tizmh/content/ $\mathrm{id} / 8536360 /$ maximized 
Dentro de la clasificación de diferentes conceptos que se engloban en este apartado, no podemos olvidar la referencia a los sujetos nativos digitales (Prensky, 2001) o, precisados posteriormente como sabios digitales (Prensky, 2009), ya que emplean la tecnología como algo cotidiano en su vida desde la infancia, haciendo de esta algo imprescindible para su día a día. Sujeto sabio digital es aquel que sabe combinar sus propias capacidades y habilidades con las potencialidades técnicas que las nuevas herramientas ofrecen, independientemente de su edad, del momento de su nacimiento o de si son personas nativas digitales (nacidas en la era de la tecnología) o inmigrantes digitales (nacidas con anterioridad al desarrollo tecnológico). Una persona sabia digital es aquel individuo joven o adulto capaz de utilizar la web 2.0 y herramientas como los blogs y las redes sociales, para aumentar su conocimiento y, en el caso de buscarlo, su audiencia. No hay duda de que estos grupos de jóvenes han crecido en una era de rápido desarrollo comunicativo y podemos presentarlos como una generación hiperconectada (Dans, 2010). Para ellos, los mundos real y virtual son dos caras de una misma moneda; on y off están integrados casi de una manera esquizofrénica (Medina, 2016). En palabras de Gutiérrez-Rubí (2016), las generaciones millennials utilizan múltiples canales y dispositivos digitales para sus actividades. Tienen un comportamiento multitasking, es decir, con capacidad (o necesidad) de hacer varias cosas a la vez; por este motivo, diferentes estudios, desde los de Dewey (1952) a Robinson (2010), señalan como comprensible que la educación, desde una perspectiva tradicional, les resulte poco motivante e incluso aburrida (Guevara, 2015).

\section{¿Qué motiva a la joven ciudadanía?}

Debemos preguntarnos: ¿innovación para mayor motivación? Según la Real Academia Española, el concepto motivación se define como ensayo mental preparatorio de una acción para animar o animarse a ejecutarla con interés y diligencia. La motivación es una fuerza que produce un concepto significativo para aquellas personas que se encargan de movilizar e influenciar a otras para actuar (familias, profesorado, coaches, líderes, influencers, youtubers o dirigentes).

Este comportamiento ha sido estudiado en el siglo XX y XXI por diferentes teorías psicológicas y pedagógicas. En sus inicios, el 
conductismo se fundamentaba en el condicionamiento simple heredado del filósofo ruso Paulov y popularizado por Watson, cuyo objetivo teórico era la predicción y el control de la conducta. Aunque siempre reconoció el rol de los factores biológicos y neurofisiológicos, puso especial énfasis en el ambiente. Así lo expresaba Watson (1930) en una conocida frase:

Dadme una docena de niños sanos y bien formados y mi mundo específico para criarlos, y yo me comprometo a tomar cualquiera de ellos al azar y entrenarlo para que llegue a ser cualquier tipo de especialista que quiera escoger. (p. 104)

El conductismo se basa en respuestas o acciones del individuo ante estímulos del ambiente, como algo externo al cerebro. Esta teoría proporciona ciertas aportaciones al ámbito de la innovación educativa ya que, si tenemos en cuenta el factor de la consecuencia (estímulo-respuesta-consecuencia) como resultado de un comportamiento, esta permitirá modificarlo produciendo un aprendizaje. Dentro de este aprendizaje se pueden esclarecer tres puntos: observación sobre qué hacen las personas participantes, bucles de realimentación (acción-alimentación-respuesta) y refuerzo, tras reforzar el estímulo se produce un aprendizaje al asociar la acción y la respuesta.

En las propuestas de innovación educativa, concretamente en el ámbito de los videojuegos y la gamificación, se pueden asociar estos tres puntos, teniendo en cuenta la retroalimentación por medio de diferentes herramientas interactivas como, por ejemplo, una barra de progresos; la propuesta que se vende como algo innovador, puede convertirse en la reproducción de una teoría pedagógica que se ha valorado como tradicional, memorística y que se aleja de la construcción personal del alumnado. Un comportamiento que puede condicionar los comportamientos a través de consecuencias; al recibir un premio tras una acción específica, se tenderá a repetir esta acción. Todo esto puede ocasionar una serie de peligros como la manipulación, tras influir en las personas para que lleven a cabo un acto. Otro de los peligros son la rutina hedonista; al actuar solo por las recompensas se puede correr el peligro de que, cuando no se reciban, no se produzcan respuestas o que se pierda la motivación y el placer por obtener dichas recompensas. No podemos olvidar también el peligro del énfasis excesivo, pretendiendo 
enfocar el sistema de gamificación solo y exclusivamente en el estatus se puede producir una pérdida de interés, ya que, por ejemplo, muchas personas no tienen la necesidad de ser reconocidas.

En contraposición con la teoría conductista, el cognitivismo se basa en que la conducta está influenciada no solo por los estímulos que hemos indicado anteriormente, sino por variables subjetivas internas; lo que importa es la interpretación que el sujeto hace de los estímulos. Muchos estudios se han dedicado a este supuesto (Ausubel, 1983; Bruner, 1973; Piaget, 1954), para estos la cognición, como acto de conocer, es el conjunto de procesos, a través de los cuales el ingreso sensorial es transformado, reducido, elaborado, almacenado, recordado o utilizado (Neisser, 1967).

Tras exponer, a grandes rasgos, las características de estas dos teorías, podemos concluir que el conductismo está relacionado con la motivación extrínseca; mientras que el cognitivismo tiene su foco en la intrínseca. En esta teoría del cognitivismo nos encontramos con la teoría de la autodeterminación (Ryan y Deci, 2000) y la facilitación de la motivación intrínseca, el desarrollo social y el bienestar estudia los factores del contexto, que amplían o reducen la motivación intrínseca, la autorregulación y el bienestar. Según esta teoría, los individuos no necesitan recompensas para motivarse.

La teoría constructivista da un paso más adelante y se centra en la creación de significados a partir de las propias experiencias de los individuos, sugiere que los sujetos aprendices crean conocimiento mientras tratan de comprender sus experiencias (Driscoll, 2000). Contrario a las teorías anteriores, el constructivismo asume que quienes aprenden no son meros recipientes vacíos que se rellenan con conocimiento, sino que están intentando crear significado activamente. En esta perspectiva, "el conocimiento no es el resultado de una mera copia de la realidad preexistente, sino de un proceso dinámico interactivo a través del cual la información externa es interpretada y reinterpretada por la mente" (Gómez y Coll, 1994, p. 54); esta acción va edificando progresivamente modelos cada vez más complejos y potentes.

En el siglo XXI surge el conectivismo que se refiere a una teoría de aprendizaje desarrollada para la era digital (Siemens, 2010), la cual ha emergido en un contexto social distinguido por la creación de valor económico a través de redes de inteligencia humana para producir conocimiento (Floridi, 2008). El aprendizaje para esta teoría puede 
residir fuera del individuo y está enfocado en conectar conjuntos de información especializada, crea un nuevo escenario, donde la tecnología posee un rol predominante porque "ha transformado los modos de hacer negocios, la naturaleza de los servicios y productos, el significado del tiempo en el trabajo, y los procesos de aprendizaje" (Fenwick 2001, p. 4). Los principios del conectivismo (Siemens, 2010) se asientan en que el aprendizaje y conocimiento se encuentran en la diversidad de opiniones como un proceso de conexión especializada de nodos o fuentes de información que pueden residir en artefactos no humanos. La capacidad para conocer más es más importante que lo actualmente conocido; alimentar y mantener las conexiones es necesario para facilitar el aprendizaje continuo. La habilidad para identificar conexiones entre áreas, ideas y conceptos es esencial; presenta la toma de decisiones como un proceso de aprendizaje en sí mismo. Seleccionar qué aprender y el significado de la información entrante es visto a través de los lentes de una realidad cambiante.

Partiendo de estas teorías, es importante destacar los tipos de motivación que existen; se atiende, así, el papel que desempeña la innovación educativa y la gamificación. De esta forma podemos distinguir la motivación intrínseca que poseen aquellas personas cuya motivación es auténtica y realizan las actividades por la satisfacción en sí que les producen. Esto está muy presente en la etapa de la infancia, ya que son seres humanos activos, curiosos, exploradores juguetones, etc. En cambio, se van dando variaciones a medida que nos desarrollamos y debido a condiciones externas. Es necesaria una serie de apoyos para el mantenimiento y la ampliación de ese estado interno.

Al respecto, una teoría clave, desarrollada por Ryan y Deci (2000), la teoría de la evolución cognitiva, tiene como propósito el estudio de aquellos factores que pueden dar explicación a la variabilidad de la motivación intrínseca. Algunos ejemplos de estos factores son la recompensa positiva, incrementadora de la motivación intrínseca; mientras que el factor de competencia no, al menos que vaya acompañado por un sentimiento de autonomía. Otros factores como las metas impuestas, las evaluaciones y las recompensas tangibles contribuyen a la inminente disminución de la motivación intrínseca. Para alcanzar este tipo de motivación deben ser cubiertas tres características basadas en las necesidades humanas: la competencia como capacidad o habilidad del individuo para realizar retos externos, la autonomía como libertad 
para decidir y actuar que desarrolla una sensación de control muy valorada, por ejemplo, en los sistemas de gamificación, y la vinculación que representa el deseo de estar conectado con otros seres.

Podemos considerar también la motivación extrínseca, que proviene de fuera del individuo y se da en aquellas personas que son controladas para ejecutar una acción. No quiere decir que esta motivación no sea autodeterminada, sino que conduce a la obtención de un resultado separable. Aquí sería conveniente señalar una segunda subteoría diseñada también por Ryan y Deci (2000): la teoría de la integración organísmica, para conocer los diferentes tipos de motivación extrínseca así como aquellos factores que promueven o impiden que esas conductas llevadas a cabo con la guía de este tipo de motivación, sean internalizadas y reguladas.

Otro tipo de motivación que podemos relacionar con la innovación educativa es la 3.0, en donde el enfrentamiento se produce entre motivación intrínseca y motivación extrínseca, recogida por Pink (2011) en su obra Drive, The Surprising Truth about what motivates us. Esta teoría parte de la premisa de que las sociedades, al igual que los dispositivos electrónicos, tienen sistemas operativos, "un conjunto de instrucciones y protocolos, en su mayoría invisibles, que permiten que todo funcione" (Teixes, 2015, p. 25). Pink (2011) clasifica la motivación en tres categorías: motivación 1.0, enfocada expresamente a la supervivencia; 2.0, estuvo vigente hasta el siglo XX, basada en castigos y recompensas y 3.0, propia de la sociedad actual, la cual exige que sea interna en los individuos para actividades menos rutinarias. Estaríamos ante una motivación intrínseca.

En el ámbito de la innovación educativa, no podemos ni pretendemos volcar todo el peso en el juego como agente único y exclusivo para mejorar la motivación del alumnado. Antes de esto, es necesario que se den y se tengan en cuenta una serie de factores que no son menos importantes como, por ejemplo, la actitud y entusiasmo del profesorado; el clima del aula; la relación entre estudiantes; los aprendizajes basados o no en la realidad, que poseen sentido y utilidad para el estudiantado (aprendizajes significativos); el reconocimiento del esfuerzo (evitando la censura y animando a la mejora), etc. El alumnado se motiva por razones diferentes y la misma actividad incentivadora produce diferentes reacciones en diferentes personas e incluso en la misma persona, en diferentes momentos. Las metodologías activas aportan variedad de 
estímulos y actividades con la frecuencia que cada estudiante o grupo precise. Hacer preguntas, prácticas, ejercicios, trabajar en grupo, cambiar de actividad, promover la participación, cambiar de lugar, etc., ayudan a captar el interés y mejorar la atención lo cual conlleva al favorecimiento de la motivación. Es decir, no nos vamos a centrar en el uso de herramientas de gamificación como los únicos instrumentos a trabajar, sino que hay que realizar un análisis exhaustivo del proceso de aprendizaje y todo lo que lo rodea, para valorar por qué el alumnado tiene una baja motivación, del tipo que sea, y qué estrategia utilizar para romper con la monotonía del aprendizaje y, desde la innovación educativa, fomentar su motivación.

\section{¿Cómo responde el sistema educativo?}

Pero, ¿cuál es la realidad de las aulas? El principal objetivo de la educación es crear una ciudadanía que sea capaz de realizar cosas nuevas, que sea creativa e innovadora, y no meramente repetidora, de generación en generación, lo que otras personas pasadas han logrado, sino ser capaces también de aportar.

Esto supone desarrollar una mente crítica que verifique y no acepte lo que se le ofrece, demanda participación y actividad de un alumnado creador y creativo. Pretende, además, que este alumnado descubra, a través de su espontaneidad, su deseo de descubrir todo lo que tiene a su alcance, lo que es capaz de hacer por su propio criterio; que sea despierto y exprese sus ideas; que responda a una necesidad innata de querer compartir todo con los demás individuos y grupos; que tome como referente en la vida una actitud dialógica (Freire, 2008; Piaget y Inhelder, 2015). Esta realidad educativa ha estado presente a lo largo de la historia, se ha enfocado desde distintas perspectivas. Según los cambios sociales en nuestra historia podemos conocer su trayectoria de forma clara, relacionada con la economía, pero aún con "problemas, desigualdades internacionales que provocaban la imposibilidad de lograr el objetivo de una educación para todos" (Carrica, 2015, p. 64).

Los seres humanos orientados al privilegio de la educación adquieren conocimientos teóricos y prácticos que los instruyen y los relacionan dentro de los sectores económicos por naturaleza global. Al profundizar en este carácter global de la educación, es necesario e imprescindible hablar de alfabetización que, aunque actualmente se hable 
del concepto de alfabetización múltiple, aún debemos seguir reflexionando en torno a lo que se ha denominado desde finales del siglo XX como alfabetización digital, relacionada estrechamente con la gamificación. En este sentido, son todo un ejemplo determinados procesos formativos llevados a cabo desde la educación superior, etapa educativa que mejor ha facilitado la "interacción con los contenidos educativos, actividad colaborativa, comunicativa y socializadora de la educación, temas estos últimos que han logrado mayor fuerza gracias al uso de las llamadas herramientas de la Web 2.0" (Chiappe, Mesa, y Álvarez, 2013, p. 2).

La educación no solo involucra comunicar lo que otros seres deben adquirir, sino que mantiene relación estrecha con el entorno social, con los medios o maneras en que se oferta y se llega a un aprendizaje con diversas interpretaciones o dimensiones (Guerrero y Faro, 2012; Pascual, 2018).

De acuerdo con las exigencias sociales actuales, se presentan una serie de retos de la educación, enfocados desde aspectos como la apertura sistemática de las escuelas a nuevas fuentes del saber, sin estrategias de censura; se apuesta por un pensamiento crítico, donde las escuelas se convierten en espacios de exploración, de descubrimiento y de invención, esto es, productoras activas de conocimiento (Pérez, 2000). Todo ello relacionado con la participación de la comunidad entera en la educación. La redefinición del rol del profesorado como entrenador, incitador, promotor, creador, mediador y educador; la aceptación de la necesidad de potenciar la alfabetización de la sociedad de la información, y la creación de nuevas comunidades educativas a partir de las escuelas actuales, como comunidades virtuales que superan el modelo fabril y desafían la renovación tecnológica. Este propósito implica una redefinición del papel del Estado en la educación con un carácter inclusivo, para luchar contra la segregación de las poblaciones más desfavorecidas mediante inversiones, una aceptación del principio de educación a lo largo de la vida, lo que proporciona diversos itinerarios del sistema y tiempos flexibles centrados en las demandas de los individuos.

Gracias al movimiento pedagógico de la Escuela Nueva (Cousinet, 1967; Decroly, 1927; Dewey, 1995; Freinet, 1976; Froebel, 1913; Kerschensteiner, 1934; Neill, 1986; Montessori, 1994; Padre Manjón, 1948; Pestalozzi, 1980; Wallon, 1987) se llevaron a cabo importantes mejoras en la concepción del alumnado distinto de la población adulta y 
con grandes capacidades imprescindibles de potenciar. Es consustancial a esta pedagogía la tensión que se podía palpar cuando se intentaba relacionar la teoría y la praxis, de modo que ninguna experiencia o reforma que se pretendiera diseñar se consideraba francamente definitiva, sino perpetuamente inacabada o imperfecta. Esta corriente de pensamiento, entre otros ámbitos, estudió la educación placentera, aportó como noción principal promulgada la idea de aprender jugando. El juego adquirió un importante protagonismo como metodología y se apostó por su introducción en los procesos educativos, ya que el profesorado vio en él algo más que un elemento de diversión o pérdida de tiempo, con potencial para aprender, desarrollar destrezas, habilidades y estrategias.

En la educación actual se plantean modelos pedagógicos más eficaces que se fijan en cómo se enseña y qué es lo que se enseña. Como hemos señalado anteriormente, a partir de concebir la edad infantil del ser humano como una etapa donde la personalidad es distinta de la de la persona adulta, el juego se proyecta como el método más apropiado para poder aprender.

La investigación educativa ha hecho aportes relevantes en lo que se refiere al estudio de los procesos de aprendizaje desde distintas variables, entre ellas "las motivaciones, autoconcepto académico, metas, elección y abordaje de tareas, habilidades cognitivas y metacognitivas, estilos y estrategias de aprendizaje, niveles de comprensión, expectativas de éxito y su influencia en el desempeño académico" (Carrascal y Sierra, 2014, p. 2). En esta perspectiva, Moreno (2011) manifiesta cómo, en la actualidad, el proceso de enseñanza se hace cada vez más complejo y el de aprendizaje se ha convertido en una experiencia mucho más desafiante para el alumnado: "cada nivel tiene su propia especificidad, la cual está determinada por las necesidades sociales y educativas a las que la escuela pretende responder y que se abrevian en los objetivos educativos para cada etapa de formación" (Moreno, 2011, p. 2).

La teoría y la práctica deben estar interrelacionadas en el ámbito educativo para generar diversos conocimientos que favorezcan la comprensión de saberes que reflejen la realidad. Es importante esta permanente relación, ya que justifica el fortalecimiento de los procesos de aprendizaje de manera inquebrantable (Álvarez, 2012). Desde las grandes figuras de la pedagogía de la Escuela Nueva hasta nuestros días se sigue demando una participación más activa del alumnado para que 
transforme aquellos procesos cotidianos, donde el diálogo debe fluir para la construcción de un feedback.

Se considera fundamental, por tanto, conocer las características de la infancia, para adaptar las metodologías más activas en las que la gamificación tiene un rol cada vez más importante, se ha pasado del homo ludens a la gamificación (Carreras, 2017). Tras encontrarnos inmersos en un entorno interconectado e hipercambiante al que debemos adaptarnos a una velocidad sin precedentes, "deberíamos asumir que la educación debiera dotar al individuo de los instrumentos cognitivos necesarios para afrontar dicho entorno" (Fernández, 2001). Los conocimientos que adquirimos en nuestra etapa de formación inicial tienen una determinada fecha de caducidad y "no podemos seguir esperando que la formación inicial nos proporcione un bagaje de conocimientos del que podamos disponer a lo largo de toda nuestra vida profesional activa" (Gros, 2004, p. 30).

Pero, ¿cómo evolucionan las instituciones educativas?, ¿estamos respondiendo a la sociedad actual?, ¿son los escenarios educativos una respuesta a las demandas del alumnado para quien fue creado? Ya en el siglo pasado Papert (1996) estableció la siguiente comparación para ejemplificar la lentitud de los cambios en las instituciones educativas. Imaginemos que hubiésemos congelado a un cirujano y a un maestro hace un siglo y ahora los trajeran de nuevo a la vida. En el caso del cirujano, este entraría en la sala de operaciones y no reconocería ni el lugar ni los objetos y se sentiría incapacitado para actuar. En cambio, el profesorado seguramente reconocería el espacio como una clase y podría encontrar una tiza y una pizarra para empezar a enseñar. La sociedad hoy exige más a profesionales de la educación y su papel cada vez se centra más en responder a una mayor velocidad, diseñando nuevos espacios y entornos de formación desde una cultura participativa.

Los equipos docentes a menudo nos encontramos con una serie de problemas a los que necesitamos aportar una solución con métodos innovadores. Pero, ¿a qué serie de problemas nos referimos? Empezando por una falta de interés en el proceso educativo, proveniente estudiantes: una apatía frente a las secuencias y cambios de actividades que pueden romper con la "zona de confort"; una falta de sentido que encuentran estudiantes en el proceso de aprendizaje, al no saber qué ganan en este, ni a qué futuro pueden optar siguiendo la cultura del esfuerzo, y un espacio y un tiempo artificiales, alejados de los procesos 
de inmersión en escenarios digitales a los que vive acostumbrado. Otro elemento para tener en cuenta en este entorno es el profesorado, el cual juega un rol muy importante dentro de la innovación. Este profesorado tiene dos papeles importantes dentro de la misma aula. El primero es ser una persona de referencia a la que el alumnado está habituado a seguir, sirve de prescriptor de las acciones y de incentivador de modelos de aprendizaje. En segundo lugar, como representante del centro educativo, es líder pedagógico y dinamizador de la organización del aula, con la capacidad de poder realizar "tanto tareas más ordinarias, como las excepcionales, que salgan fuera de la rutina, y de alguna forma que puedan también condicionar la capacidad de aplicación con éxito de la gamificación en el aula" (Contreras y Eguia, 2016, p. 14). A la desmotivación que podemos percibir en el profesorado y que impide desarrollar prácticas de innovación educativa, podemos unir su falta de reconocimiento social y de apoyo de las familias, la falta de recursos personales, materiales, digitales y económicos, que convierten las aulas de la enseñanza formal en espacios donde "se hace lo que se puede" y se intenta responder a las necesidades del alumnado.

\section{¿Puede la tecnología ayudarnos a motivar a nuestro alumnado? Experiencias}

La nueva realidad social de nuestros días cuestiona cada vez más la posición que los sistemas educativos están teniendo en cuanto en la incorporación de la innovación en las aulas y, más concretamente, de dispositivos digitales o simuladores de juego en las prácticas pedagógicas que se asientan en la gamificación. La concepción de los juegos contra los que luchaba la Escuela Nueva y que, actualmente diferentes sectores los siguen viendo como "antítesis del aprendizaje" (Contreras, 2016, p. 27), se ha ido dejando a un lado, al surgir con fuerza las experiencias de gamificación (Rengifo, Smith, Jara, Oswaldo, y Muñoz, 2017), que crean espacios donde se construyen roles, identidades y lenguaje en los que se refuerzan los estereotipos preconcebidos o, por el contrario, se rechazan con la obtención de información contraria o alternativa. El juego y el aprendizaje tienen varios aspectos en común: el afán de superación, la práctica y el entrenamiento que conducen al aumento de las habilidades o capacidades, la puesta en práctica de 
estrategias que conducen al éxito y ayudan a superar las dificultades de la vida cotidiana (Sánchez, 2010).

Otro tipo de visión basada en la herencia de la ética protestante del trabajo defiende que el aprendizaje, al igual que otra labor, debe realizarse como si fuera un "deber", organizando y estructurando el tiempo y evitando la introducción de elementos que alteren dicho tiempo. Es más, apunta que toda diversión y entretenimiento debe dejarse para los momentos de ocio y tiempo libre (Himanen, 2002). Escribano (2013) va más allá al atreverse a diferenciar entre gamificación y ludictadura. Establece este último concepto como un estado que se da en nuestra sociedad actual de manera que lo que algunos denominan gamificación "se aplica de forma vertical, desde arriba hacia abajo y no como quizá deberían generarse las reglas de todo juego social: de forma democrática, entre todos y desde abajo hacia arriba" (Escribano, 2013, p. 67). Esta gamificación forzosa a la que se refiere este autor induce a comportamientos deseados sin discutir los "por qué" o sin permitir que participen en las reglas del juego todos los sujetos implicados.

Analizando los resultados que se extraen tras un estudio sobre los efectos de la gamificación en el aula, titulado Assessing the effects of gamification in the classroom: a longitudinal study on intrinsic motivation, social comparison, satisfaction, effort and academic performance (Hanus y Fox, 2015), podemos extraer de él tres conclusiones aplicables específicamente al diseño gamificado que se creó para llevar a cabo esta investigación. La primera de ellas hace referencia a que estudiantes del curso gamificado resultaban tener menos motivación, menos empoderamiento y menos satisfacción con el tiempo. Otra conclusión se relacionaba con el curso gamificado y cómo afectó negativamente las calificaciones finales a través de la motivación intrínseca. Finalmente, la tercera conclusión, se refería a que los sistemas gamificados ofrecen fuertes recompensas que pueden tener efectos negativos. McGonigal (2011), diseñadora de numerosos juegos, en su libro Reality is Broken, intenta evitar el uso de la palabra gamification y utiliza conceptos de esta categoría epistémica, llevándola más allá y relevando así su importancia oculta. Plantea que la realidad está rota, que "en la sociedad actual, videojuegos y juegos de computadora están cumpliendo necesidades humanas genuinas que el mundo real actualmente no puede satisfacer" (McGonigal, 2011, p. 4). 
A partir de la reflexión sobre lo expuesto, los enfoques y modelos educativos no responden a los nuevos retos sociales de cambio acelerado y cultura digital (Siemens, 2010). Es importante construir nuevos enfoques que dejen atrás la sociedad de la información y se adentren en la sociedad del conocimiento, considerando al ser humano en su triple dimensión: individual, social y ambiental (Tobón, 2013). Por ello, se debe lograr un acuerdo en los ejes de la sociedad del conocimiento, base para avanzar en nuevas perspectivas educativas. Los procesos de aprendizaje han de convertirse en una enseñanza educativa desde los distintos agentes, "no se trata de transmitir el puro saber, sino una cultura que permita entender nuestra condición y ayudarnos a vivir. El reto de la globalidad es también el reto de la complejidad" (Morin, 1999, p. 11). Por ello, es necesario enfrentar a las generaciones más jóvenes al conocimiento sin mediaciones en un contexto escolar que represente al mundo real.

Actualmente, la tecnología ha desarrollado mejores medios innovadores para llegar a mayor número de personas, las llamadas tecnologías emergentes. Ramírez (2014) las califica como tecnologías disruptivas e innovaciones basadas en la ciencia con la capacidad de transformar una industria o crear una nueva. Abarcan las telecomunicaciones, los dispositivos móviles, la realidad aumentada, los videojuegos, la gamificación, el m-learning, el flipped classroom y los cursos masivos, abiertos y en línea (Osuna-Acedo y Gil-Quintana, 2017), entre otros.

Parece una evidencia que la mayor parte de la niñez se inicia en el mundo de la informática a través de los videojuegos. Por ello, cuando utiliza el ordenador o usa otros programas informáticos (procesadores de texto, gráficos...), tiene ya ciertos conocimientos y destrezas adquiridas fuera del ámbito escolar. Es decir, a través del uso de los videojuegos se produce buena parte de la alfabetización en la infancia. Este hecho tiene dos consecuencias importantes, según Gros (2004); por un lado, al parecer los niños y las niñas adquieren unos conocimientos "naturales" sobre el uso de las tecnologías, lo que lleva a pensar erróneamente que saben mucho más sobre este tema que incluso las personas adultas y, por ello, no parece necesario dedicar tiempo a facilitar un conocimiento que ya poseen. Por otro, la escuela desaprovecha estos conocimientos sobre las tecnologías adquiridos en otros contextos no formales y, cuando se trata del uso de estas en el entorno escolar, se centra en algo que el alumnado ya sabe: los aspectos instrumentales. 
Salomón y Globerson (1992), ya en el pasado siglo XX, realizaron una interesante distinción entre los efectos de aprender con la tecnología y los efectos de la tecnología; el primer tipo se refiere, por ejemplo, a los efectos que la utilización del ordenador para una actividad en concreto tiene en la persona (conocimientos específicos y estrategias); mientras que el segundo tipo hace referencia a los "posos" cognitivos o consecuencias que el uso de la tecnología representa más adelante. Esto se traduce en aprendizajes propios de la cultura de la sociedad de la información, que tendrá consecuencias a largo plazo.

El uso de la tecnología y, más concretamente desde el ámbito de la gamificación en el aprendizaje, es una realidad y favorece dos aspectos fundamentales de los procesos educativos actuales (Foncubierta y Rodríguez, 2014). En primer lugar, posibilita la ampliación y prolongación de los espacios de aprendizaje y lleva el acceso a los contenidos más allá del aula. El estudiantado puede trabajar sobre el contenido en concreto en cualquier momento y desde cualquier lugar siempre que tenga acceso a internet. En segundo lugar, este estudiantado está acostumbrado a consumir y producir contenidos digitales, por lo que el uso de la tecnología hace que el contenido sea más familiar e intuitivo. Los dispositivos móviles permiten que se tenga en cuenta el diseño de estos contenidos para que se pueda acceder a ellos desde este tipo de dispositivos. Además, gracias a esto, se automatizan los procesos como asignación de emblemas, puntos, bienes virtuales... lo cual hace la gamificación más atractiva.

Los dispositivos electrónicos son herramientas que se deben aprovechar para desarrollar procesos de aprendizaje. Partiendo de esta realidad, podemos encontrar experiencias de gamificación en la creación de escenarios digitales (Reyes, Granados, Sánchez, y Melchor, 2017) en los MOOC (Gil-Quintana, Camarero-Cano, Cantillo-Valero, y Osuna-Acedo, 2018), como apoyo al personal docente, como herramienta de trabajo para la orientación psicopedagógica (Planchart, Reina, Padrón, Montero, y Ferrer, 2018), para facilitar el aprendizaje (Arnaiz, 2017; Prieto, 2018), para la motivación (Rodríguez, Moreno y Vera, 2017), para desarrollar habilidades cognitivas y destrezas motrices en estudiantes (Sánchez, 2014), para construir la convivencia escolar (Causado y Pacheco, 2018), como herramienta de evaluación formativa (Díaz, 2017) y como estrategia que fomenta el pensamiento crítico a través de los roles jugadores, creadores y diseñadores de 
experiencias lúdicas de aprendizaje (Almonte y Bravo, 2016; Soler, Contero y Alcañiz, 2017).

No todos los autores o autoras que se inmiscuyen en la tarea de tratar la gamificación hablan de ella positivamente, resaltando sus ventajas y potencial. Existen muchas críticas hacia este novedoso ámbito, que tachan esta práctica de ser defectuosa y engañosa, de utilizar estrategias artificiales y aburridas, de falta de narratividad y voluntariedad. Comenzaremos desde esta línea de pensamiento por señalar el achaque que relaciona la gamificación con el atribuir al alumnado demasiados ingredientes lúdicos que conducen a que, en el futuro, no sea capaz de valerse de su fuerza de voluntad sin estos apoyos. Esta teoría puede ser altamente apoyada. No obstante, también hemos encontrado interesante resaltar la idea que "la voluntad se refuerza ante todo conquistando metas, ganando en la lucha diaria por nuestros objetivos, pero también limando las barreras y haciendo que las tareas sean más fáciles de disfrutar" (Rodríguez y Santiago 2015, p. 15). Por otro lado, hay quien critica también la gamificación y otras innovaciones educativas defendiendo que se utiliza solo y exclusivamente por su factor novedoso, olvidando que "no hay nada nuevo bajo el sol de Platón, que lo inventó todo en sus Diálogos ... que no hay nada mejor para tratar cualquier tema, natural o social, que un buen debate" (Navajas, 2018). Se critica el factor de competición que se genera en estos sistemas, ciertos problemas atribuidos como comportamientos no éticos, y la poca cooperación y colaboración (Vaibhav y Gupta, 2014).

\section{Conclusión}

Nadie puede dudar que vivimos en una sociedad hipercambiante, en continua demanda y exigencia a los individuos que conviven en ella. Unas personas nacen y aprenden ciertas habilidades de manera subconsciente y otras deben adquirirlas una vez en su adultez, de una forma plenamente consciente y con las herramientas necesarias para ello. Esta característica hace que el profesorado de nuestros centros educativos deba estar al tanto de las propuestas innovadoras y de las necesidades de las nuevas generaciones, apostando por el cambio y la actualización de las prácticas pedagógicas. Surgieron en su momento las tecnologías y con ellas un giro a la enseñanza en las aulas para dar respuesta a la población nativa digital; ahora estamos al tanto del surgimiento de la 
gamificación a través de nuevos paradigmas, dimensiones alternativas que pueden ayudarnos a captar la motivación de los individuos que llegan a la clase y se encuentran con una realidad bastante menos parecida a la que viven fuera de ella, pues interactúan de forma continuada a través de sus dispositivos móviles. Nuestra labor como docentes está en optar por la práctica que más se ajuste a las capacidades de nuestro alumnado, fomentando su motivación y despertando en él las ganas de aprender socializándose con sus semejantes.

Este ensayo ha pretendido hacer al personal investigador educativo consciente de las reflexiones que nos hacemos docentes, con el propósito de ayudar a reflexionar sobre la situación en la que nos encontramos tanto a nivel general en la sociedad como a nivel educativo. De esta forma, aportamos nuestro granito de arena para poder seguir con otros estudios, en los que se pretenda esclarecer para qué sirve la gamificación en la educación y qué podemos hacer con esta tendencia en estos momentos, ante las nuevas situaciones sociales y el estudiantado inmerso activamente en la sociedad digital. Analizar los tipos de motivaciones y cómo se desarrolla cada uno es muy conveniente para descubrir de qué manera están motivados los equipos docentes y qué debemos hacer para generar en ellos el tipo de motivación que deseamos, al ser sujetos mediadores fundamentales entre el alumnado y su aprendizaje. La motivación es de especial importancia en los centros educativos para captar la atención y conseguir un aprendizaje significativo; si los grupos estudiantiles están más motivados fuera del aula que en ella, no lograremos un proceso de aprendizaje rico y pleno y, los espacios de educación no formal nos estarán dando de nuevo una lección en el desarrollo de las capacidades.

No hay duda, tampoco, de que nuestras herramientas aliadas para conseguir esto son los dispositivos electrónicos y las redes sociales, que otorgan la posibilidad de abrir caminos para llegar hasta nuestro alumnado de una manera u otra. No podemos olvidar que debemos tener especial cuidado en cómo las usamos, olvidando el cacharreo tecnológico que pretende enseñar lo que el alumnado ya conoce, sino, más bien, dándole un sentido pedagógico que considere la simple integración de las tecnologías de la información y la comunicación (TIC), para dar paso a las tecnologías de la relación y la comunicación (TRIC) y, de forma complementaria, a las tecnologías para el aprendizaje y el conocimiento (TAC). 
Aún tenemos un largo camino por recorrer en el ámbito de la investigación educativa, con el fin de consolidar unas premisas concretas que nos ayuden a encaminar correctamente el ámbito de la gamificación en las aulas y la relación que este planteamiento puede tener con las tecnologías digitales. Sigamos adelante con el fin de edificar, sobre una base sólida, una educación motivante de todas las personas y para todas ellas.

\section{Referencias}

Almonte, M. y Bravo. J. (2016). Gamificación y e-learning: estudio de un contexto universitario para la educación de su diseño. Tecnología, Ciencia y Educación, 4, 52-62. Recuperado de https://bit. ly/2riUbRN

Álvarez, C. (2012). La relación teoría-práctica en los procesos de enseñanza-aprendizaje. Education siglo XXI, 1. Recuperado de https://bit.ly/2FDvjto

Arnaiz Martín, J. M. D. (2017). Gamificación de FarolApp. Proyecto Fin de Carrera / Trabajo Fin de Grado, E.T.S. de Ingenieros Informáticos (UPM), Madrid.

Ausubel, N.H. (1983). Psicología educativa: Un punto de vista cognoscitivo. México: Editorial TRILLAS.

Bauman, Z. (2013). Sobre la educación en un mundo líquido: Conversaciones con Ricardo Mazzeo. Buenos Aires: Paidós.

Beck, J. y Wade, M. (2004). Got game: How the gamer generation is reshaping business forever. Boston: Harvard Business School Press.

BBC (2018). Creo que nunca hice una llamada de teléfono: Cómo se comunica la generación muda. BBC Mundo. Recuperado de https://bit.ly/2E0vge4

Bruner, J. S. (1973). The relevance of education. New York: Norton.

Carrascal, S. y Sierra, I. (2014). Influencia de los contextos de enseñanza en la calidad de aprendizaje universitario. Redalyc, 2. Recuperado de https://bit.ly/2FE49m5

Carreras, C. (2017). Del homo ludens a la gamificación. Quaderns de filosofia i ciència, 4(1), 107-118.

Carrica, S. (2015). La educación para el desarrollo (Tesis doctoral). Universidad de Navarra. 
Cuasado Escobar, R. y Pacheco Bohórquez, M. L. (2018). El aprendizaje basado en videojuegos y la gamificación como estrategias para construir y vivir la convivencia escolar. Revista CEDOTIC, 3(1). Recuperado de https://bit.ly/2Q5K4yv

Chiappe, A., Mesa, N., y Álvarez, C. (2013). Transformaciones en las concepciones de los docentes de educación secundaria acerca de la Web 2.0 y su uso en los procesos de enseñanza. Scielo, 2. Recuperado de https://bit.ly/2HKnZSC

Colás Bravo, P. (2003). Internet y aprendizaje en la sociedad del conocimiento. Revista Científica de Comunicación y Educación (Comunicar), 20, 31-35. Recuperado de https://bit.ly/2w7z4rn

Contreras Espinosa, R. (2016). Juegos digitales y gamificación aplicados en el ámbito de la educación. Revista Iberoamericana de Educación a Distancia, 19(2), 27-33. Recuperado de https://bit. ly/2Ib8HFW

Contreras, R. S. y Eguia, J. L. (2016). Gamificación en aulas universitarias. Barcelona: Universidad Autónoma de Barcelona.

Cousinet, R. (1967). La escuela nueva. Barcelona: Editorial Luis Miracle.

Castells, M. (2000). Internet y la sociedad red. Conferencia de Presentación del Programa de Doctorado sobre la Sociedad de la Información y el Conocimiento. Universitat Oberta de Catalunya.

Dans, E. (2010). Todo va a cambiar: Tecnología y evolución: Adaptarse o desaparecer. Barcelona: Deusto Ediciones.

Decroly, O. (1927). La función de la globalización y la enseñanza. Revista de Pedagogía, Madrid. Recuperado de https://bit.ly/2rrPPrO

Dewey, J. (1952). La busca de la certeza: Un estudio de la relación entre el conocimiento y la acción. Fondo de Cultura Económica.

Dewey, J. (1995). Democracia y educación. Madrid: Ediciones Morata.

Díaz, P. P. (2017). Gamificando con Kahoot en evaluación formativa. Revista Infancia, Educación y Aprendizaje, 3(2), 112-117.

Driscoll, M. (2000). Psychology of Learning for Instruction. Needham Heights, MA: Allyn \& Bacon.

Escribano, F. (2013). Gamificación versus ludictadura. Obra digital, 5, 58-72. Recuperado de https://bit.ly/2jofy0w

Fenwick, T. (2001). Tides of change. New themes and questions in workplace learning. In T. Fenwick (Ed.), Socio-cultural perspectives on learning through work (pp. 3-17). San Francisco: Jossey Bass. 
Fernández, A. (2012). Millennials: La generación malcriada que quiere cambiar el mundo. Periódico ABC. Madrid. Recuperado de https://bit.ly/lheABKd

Fernández Hermana, L. A. (2001). Alfabetización digital obligatoria. Recuperado de https://bit.ly/2HPdWHY

Floridi, L. (2008). Glossary of term for the digital era. University of Hertfordshire \& University of Oxford. Recuperado de https://bit. ly/2I5nn9F

Foncubierta, J. M. y Rodríguez, J. M. (2014). Didáctica de la gamificación en la clase de español. Ediciones Edinumen. Recuperado de https://bit.ly/1XfTVPK

Freinet, C. (1976). Las invariantes pedagógicas. Barcelona: Editorial Laia.

Freire, P. (2008). Pedagogía del oprimido. Buenos Aires: Siglo XXI Editores.

Froebel, F. (1913). La educación del hombre. Madrid: Daniel Jorro, Editor.

García Jiménez, A., Tur-Viñes, V., y Pastor Ruiz, Y. (2018). Consumo mediático de adolescentes y jóvenes. Noticias, contenidos audiovisuales y medición de audiencias, ICONO 14. Recuperado de https://doi.org/10.7195/ri14.v16i1.1101

García Sánchez, M. R., y Godínez Alarcón, G. (2015). Sociedad del conocimiento frente a la desigualdad social. $4^{\circ}$ Congreso Virtual Internacional sobre Tecnología, Educación y Sociedad (pp. 1-12).

Gil-Quintana, J. (2015). Narrativa digital e infancia: Es la hora de la generación CC. Revista Mediterránea de Comunicación, 7(1), 79-90. Recuperado de https://doi.org/10.14198/MEDCOM2016.7.1.5

Gil-Quintana, J. (2016). Interconectado apostando por la construcción colectiva del conocimiento. Aprendizaje móvil en la educación infantil y primaria. Revista de medios y educación, 54, 185-203. Recuperado de https://bit.ly/2Kw3qqV

Gil-Quintana J., Camarero-Cano L., Cantillo-Valero C., y Osuna- Acedo S. (2017). sMOOC and Gamification - A Proposed Ubiquitous Learning. In Wu TT., Gennari R., Huang YM., Xie H., Cao Y. (Eds) Emerging Technologies for Education. Lecture Notes in Computer Science, 10108. Springer, Cham. 
Gobierno de España, Ministerio de Economía y Competitividad. (s. f.). Plan Estatal de Investigación Científica y Técnica y de Innovación 2013-2016. Recuperado de https://www.upf.edu/web/medium/projectes-de recerca//asset_publisher/hNFMvM6Tizmh/ content/id/8536360/maximized

Gómez, C. y Coll, C. (1994). De qué hablamos cuando hablamos de constructivismo. Cuadernos de Pedagogía, 221, 54-58. Constructivismo. Barcelona: Fontalba.

Gros, B. (2004). Pantallas, juegos y educación. La alfabetización digital en la escuela. Bilbao, Desclée De Brouwer

Guerrero, J., y Faro, T. (2012). Breve análisis del concepto de educación superior. Alternativas en Psicología, XVI (27), 34-41. Recuperado de https://bit.ly/2JOIABJ

Guevara, J. M. (2015). Press Start, los videojuegos como recurso educativo: Una propuesta de trabajo con Minecraft y ciencias sociales.Ar@cne, Revista Electrónica de Recursos en Internet sobre Geografia y Ciencias Sociales. Recuperado de https://bit. ly/2HMtNHi

Gutiérrez-Rubí, A. (2015). La generación millennial y la nueva política. Revista de Estudios de Juventud, 108, 161-169. Recuperado de https://bit.ly/2HS5qYQ

Gutiérrez-Rubí, A. (2016). 6 rasgos clave de los millennials, los nuevos consumidores, en Forbes México. México.

Hanus, M. D., y Fox, J. (2015). Assessing the effects of gamification in the classroom: A longitudinal study on intrinsic motivation, social comparison, satisfaction, effort, and academic performance. Computers \& Education, 80, 152-161. Recuperado de https://bit. ly/2DiaTK 8

Himanen, P. (2002). La ética del backer y el espiritu de la era de la información. Barcelona: Destino.

Kerschensteiner, G. (1934). Concepto de escuela del trabajo. Madrid: Ediciones la Lectura.

Krüger, K. (2006). El concepto de sociedad del conocimiento. Revista Bibliográfica de Geografia y Ciencias Sociales, 11, 683. Recuperado de https://bit.ly/1qB05X3

Lessig, L. (2012). Remix. Cultura de la remezcla y derecho de autor en el entorno digital. Barcelona: Icaria. 
Manjón, A. (1948). Modos de enseñar. Alcalá de Henares: Imprenta Talleres Penitenciarios.

Marta-Lazo, C., y Gabelas-Barroso, J. A. (2016). Comunicación digital. Un modelo basado en el factor relacional. Barcelona: Editorial UOC.

McGonigal, J. (2011). Reality is broken: Why games make us better and how they can change the world. Estados Unidos de América: Penguin Books

Medina S. (2016). Los millennials su forma de vida y el streaming. Gestión y estrategia, 50, 121-137. Recuperado de https://bit. $1 \mathrm{y} / 2 \mathrm{jrNdXt}$

Montessori, M. (1994). Ideas generales sobre el método. Manual práctico. Madrid, España, CEPE: Colección clásicos CEPE.

Moreno, T. (2011). Didáctica de la educación superior: Nuevos desafíos en el siglo XXI. Perspectiva educacional, 2. Recuperado de https://bit.ly/2HMwqNa

Morin, E. (1999). La tete bien faite. París: Seuil.

Navajas, S. (2018). Eso no estaba en mi libro de historia de la filosofía. Editorial Almuzara.

Neill, A. S. (1986). Summerhill. Un punto de vista radical sobre la educación de los niños. México: Fondo de Cultura Económica.

Neisser, U. (1967). Cognitive psychology. Appleton-Century-Crofts. New York

Osuna-Acedo, S., y Gil-Quintana, J. (2017). El proyecto europeo ECO. Rompiendo barreras en el acceso al conocimiento. Educación $X X, 20(2), 189-213$. Recuperado de https://bit.ly/2I96TtV

Papert, S. (1996). The connected family: bridging the digital generation gap. Atlanta: Longstreet Press.

Pascual, J. (2018). La disparidad de género en los medios digitales. En Cantillo-Valero y Gil-Quintana, Comunicación y desarrollo en la sociedad digital: Nuevos discursos y viejos valores del poder cultural (pp. 31-42). Sevilla: Egregius.

Pérez Tornero, J. M. (2000). Comunicación y educación en la sociedad de la información. Barcelona: Paidós.

Pestalozzi, J. H. (1980). Cartas sobre la educación de los niños. México: Porrúa.

Piaget, J. (1954). The construction of reality in the child. NY: Basic Book. 
Piaget, J., y Inhelder, B. (2015). Psicología del niño. Edición, prólogo e indices de Juan Delval. Madrid: Ediciones Morata. Recuperado de https://bit.ly/2FDHmqt

Pink, D. (2011). Drive. Edimburgo: Canongate Books.

Planchart, S. C. F., Reina, M. F., Padrón, N. D. P., Montero, M. E. M., y Ferrer, E. E. C. (2018). La gamificación como herramienta en el trabajo docente del orientador: Innovación en asesoramiento vocacional desde la neurodidáctica. Revista Iberoamericana de Educación, 78(1), 165-182.

Prensky, M. (2001). Nativos e inmigrantes digitales. Institución Educativa SEK. Recuperado de https://bit.ly/2w6wfW6

Prensky, M. (2009). H. sapiens digital: From immigrants and digital natives to digital wisdom. Innovate 5 (3). Recuperado de https:// bit.ly/2FEZH6u

Prieto, E. (2018). Gamificación, motivación y aprendizaje en educación primaria (Tesis de maestría). Madrid: UNED.

Ramírez, M. (2014). Tecnologías emergentes en el movimiento educativo abierto. Monterrey, México: Tecnológico de Monterrey.

Reyes, J. A. G., Granados, S. A. O., Sánchez, E. G., y Melchor, I. G. F. (2017). Propuesta de gamificación en el aula: Uso de una plataforma para motivar a los estudiantes del Programa Académico de Informática de la Universidad Autónoma de Nayarit. EDUCATECONCIENCIA, 13(14).

Rengifo, P., Smith, Y., Jara, V., Oswaldo, E., y Muñoz, J. I. (2017). Experiencias motivacionales gamificadas: Una revisión sistemática de literatura. Innovación educativa (México, DF), 17(75), 63-80.

Robinson, K. (2010). ¡A iniciar la revolución del aprendizaje! TED. Recuperado de https://bit.ly/2DXDyDs

Rodríguez, F. y Santiago, R. (2015). Gamificación: Como motivar a tu alumnado y mejorar el clima en el aula. Innovación Educativa. Madrid: Digital-Text. Grupo Océano. Recuperado de https://bit. ly/2js8uQG

Rodríguez, R. A., Moreno, E. J., y Vera, P. M. (2017). Un enfoque motivacional por medio de la gamificación en el ámbito universitario. $V$ Congreso Internacional de Videojuegos y Educación (CIVE 2017). Recuperado de https://bit.ly/2DPkV5f

Ryan, R. y Deci, E. (2000). Self-determination theory and the facilitation of intrinsic motivation, social development, and well-being. 
American Psychologist, 55(1), 68-78. Recuperado de https://bit. $1 \mathrm{y} / 2 \mathrm{bC} 751 \mathrm{E}$

Salomón, G. y Globerson, T. (1992). Co-participando en el conocimiento: la ampliación de la inteligencia humana con las tecnologías inteligentes. Comunicación, lenguaje y educación, 13, 6-22. Recuperado de https://bit.ly/2HQtfjJ

Sánchez Benítez, G. (2010). Las estrategias de aprendizaje a través del componente lúdico. Suplementos Marco ELE, 11. Recuperado de https://bit.ly/2Kw6F $1 \mathrm{z}$

Sánchez, M. D. (2014). Metodologías activas y aprendizaje por descubrimiento. Las TIC y la educación. Chile: Marpadal Interactive Media.

Semana (2017). Así son los Peter Pan del siglo XXI en psicología. Recuperado de https://bit.ly/2Ag7Hc4

Siemens, G. (2010). Conectivismo: Una teoría de aprendizaje para la era digital. En Aparici (Ed.), Conectados en el ciberespacio (pp. 77-89). Madrid: UNED.

Sinek, S. (2017). Simon Sinek. Recuperado de https://bit.ly/2BlB15D

Soler J. L., Contero M. y Alcañiz M. (2017). VR Serious Game Design Based on Embodied Cognition Theory. En, Alcañiz M., Göbel S., Ma M., Fradinho Oliveira M., Baalsrud Hauge J., Marsh T. (Eds.), Serious Games. JCSG 2017. Lecture Notes in Computer Science, 10622. Cham: Springer.

Stein, J. (2013). Millennials: The Me Me Me Generation. Time. Recuperado de https://ti.me/lizflyq

Tapscott, D. (1998). Growing up digital. The rise of the net generation. New York: McGraw-Hill.

Tedesco, J. C. (1999). Educación y sociedad del conocimiento y de la información. Memorias del Encuentro Internacional de Educación Media. Bogotá: Secretaría de Educación de Bogotá. Recuperado de https://bit.ly/2rm9oBY

Teixes, F. (2015). Gamificación, motivar jugando. Barcelona: Editorial UOC.

Tobón, S. (2013). Formación integral y competencias. Pensamiento complejo, currículo, didáctica y evaluación. Bogotá: ECOE.

Tobón, S. (2014). Proyectos formativos: Teoría y práctica. México: Pearson. 
Universitat Pompeu Fabra. (s. f.). Transalfabetismos. Competencias, transmedia y estrategias informales de aprendizaje de los adolescentes. Recuperado de https://www.upf.edu/web/medium/projectes-de-recerca/-/asset_publisher/hNFMvM6Tizmh/content/ id/8536360/maximized\#.XIW7rpwzbIX

Vaibhav, A. y Gupta, P. (2014). Gamification of MOOCs for increasing user engagement. In MOOC, Innovation and Technology in Education (MITE), 290-295.

Wallon, H. (1987). Psicología y educación del niño. Una comprensión dialéctica del desarrollo y la educación infantil. Madrid, Visor-Mec.

Watson, J. B. (1930). Behaviorism. New York: Norton. 
